\title{
Molecular Analyses of Biofilm-Producing Clinical Acinetobacter baumannii Isolates from a South Indian Tertiary Care Hospital
}

\author{
Balaram Khamaria Manmath Lama ${ }^{a} \quad$ Chanakya Pachi Pulusu ${ }^{a}$ \\ Amarendra Pratap Biswal ${ }^{a}$ Sai Manoz Lingamallu ${ }^{a}$ Bhargava Sai Mukkirla ${ }^{a}$ \\ Amit Kumar Sahoo ${ }^{a}$ Harioum Sambhu Narayan Dash ${ }^{a}$ Rewa Shardab \\ Prakash Kumar $^{\text {b Eswarappa Pradeep Bulagonda }}{ }^{a}$ \\ aDepartment of Biosciences, Sri Sathya Sai Institute of Higher Learning, Andhra Pradesh, India; \\ ${ }^{b}$ Department of Microbiology, Sri Sathya Sai Institute of Higher Medical Sciences, Andhra Pradesh, India
}

\section{Highlights of the Study}

- The emergence of colistin-resistant Acinetobacter baumannii led to analysis of clinically isolated $A$. baumannii.

- Isolates collected over 6 months were analysed for biofilm production, genes associated with antibiotic resistance and virulence, and mobile genetic elements (MGEs).

- Strict implementation of infection control guidelines is critical to combat the spread of antimicrobial resistance.

\section{Keywords}

A. baumannii $\cdot \operatorname{Tn} 1213 \cdot$ AbaR (Acinetobacter baumannii antibiotic resistance) island $\cdot$ Biofilm

\begin{abstract}
Objectives: The aim of the study was to determine the presence of antimicrobial-resistance (AMR) genes, virulence genes, and mobile genetic elements (MGEs) in 14 biofilmproducing clinical isolates of Acinetobacter baumannii. Materials and Methods: PCR amplification was performed to analyse the prevalence of genes associated with antibiotic resistance (extended-spectrum $\beta$-lactamases [ESBLs] and metallo- $\beta$-lactamases [MBLs]), virulence factors, MGEs (class 1 integron, Tn1213, and A. baumannii antibiotic resistance
\end{abstract}

karger@karger.com www.karger.com/mpp

Karger $\stackrel{\text { ' }}{5}$

GOPEN ACCESS
(C) 2020 The Author(s)

Published by S. Karger AG, Basel

This is an Open Access article licensed under the Creative Commons Attribution-NonCommercial-4.0 International License (CC BY-NC) (http://www.karger.com/Services/OpenAccessLicense), applicable to the online version of the article only. Usage and distribution for commercial purposes requires written permission.
[AbaR]), and comM among the study isolates. Random amplified polymorphic DNA (RAPD) PCR was then deployed to understand their phylogenetic relationship. All the isolates were investigated for biofilm production. Results: Two isolates were antibiotic-sensitive (AS), 3 were multi-drug-resistant (MDR), and the remaining 9 were extensively drug-resistant (XDR). The majority of the isolates were found to be positive for biofilm production and were sensitive against tetracycline and colistin only. Ab14 and Ab11 were found to be resistant to minocycline and colistin, respectively. bla TEM, bla $a_{\mathrm{OXA}}, b / a_{\mathrm{NDM}}, b / a_{\mathrm{VIM}}, b / a_{\mathrm{SIM}}$, and bla $a_{\mathrm{PER}-1} ;$ class 1 integron; composite transposon Tn1213; AbaR island, and virulence factor genes were detected among the isolates. These pathogens were found to have originated from multiple clonal lineages. Conclusion: Biofilm-producing A. baumannii with 
multiple virulence and AMR genes pose serious clinical challenges. The presence of MGEs further compounds the situation as these isolates serve as potential reservoirs of AMR and virulence genes. Together with their capacity for natural competence, A. baumannii, if left unchecked, will lead to the spread of resistance determinants to previously sensitive bacteria and may aid in the emergence of untreatable pandrug-resistant phenotypes.

(C) 2020 The Author(s)

Published by S. Karger AG, Basel

\section{Introduction}

Acinetobacter baumannii causes a variety of community-acquired and nosocomial infections of the cerebrospinal fluid, lungs, wounds, urinary tract, skin, soft tissue, and bloodstream, with high rates of morbidity and mortality [1-3]. A. baumannii topped the WHO (World Health Organisation) list of 12 bacteria for which new drugs are urgently required [4]. Irrational use of antibiotics and selective pressure has led to A. baumannii emerging as an antibiotic-resistant pathogen in both hospital and environmental settings. An increased prevalence of multi-drug-resistant (MDR) and extensively drug-resistant (XDR) strains of $A$. baumannii have been observed in large numbers over the past 2 decades [5]. These pathogens are found to persist over extended periods of time on inanimate surfaces under a wide range of environmental conditions, largely due to their capacity to produce strong biofilms [6]. Scant information is available with regard to the clonal relatedness and diversity of $A$. baumannii strains on the Indian subcontinent. Genomic analysis of MDR A. baumannii has indicated the presence of integrons, transposons, and other mobile genetic elements (MGEs) such as A. baumannii antibiotic resistance $(\mathrm{AbaR})$ islands. Acquisition of these resistance determinants has been attributed to the potential for horizontal gene transfer among A. baumannii isolates $[7,8]$. The ATPase-encoding gene comM (competence protein) is split by the incoming AbaR island, leading to the formation of 2 segments, i.e., comM-AbaR and AbaR-comM [9]. Tn1213 is a composite transposon made up of up two distinct insertion sequences, ISPa12 and ISPa13 [10]. An extended-spectrum $\beta$-lactamase (ESBL), bla $a_{\mathrm{PER}-1}$, is generally ferried by this transposon across the genomes [11]. Furthermore, these pathogenic bacteria are reported to harbor several virulence factors that help in quorum sensing and regulation, i.e., adherence, drug efflux, biofilm formation, and serum resistance. They also help the pathogen to colonize the host, evade or suppress the host's immune response, obtain essential nutrients and growth factors from the host, and gain entry or exit from the host.

In this study, colistin-resistant (MDR) A. baumannii was isolated from the blood collected at central line of a patient operated for coronary artery bypass at the Sri Sathya Sai Institute of Higher Medical Sciences, Prasanthigram (SSSIHMS-PG). This prompted the Infection Control Board to investigate A. baumannii infections over a period of 6 months, from October 2015 to March 2016. Here, a total of 14 clinically isolated A. baumannii (2 antibiotic-sensitive [AS], $3 \mathrm{MDR}$, and 9 XDR isolates) were analysed for the prevalence of virulence factors and antibiotic resistance genes. The isolates were then screened for the presence of composite transposon $\operatorname{Tn} 1213$, bla $_{\mathrm{PER}-1}$, and the host gene comM to understand their genetic context. All the isolates were investigated for their capacity to produce biofilms.

\section{Materials and Methods}

\section{Setting}

The study included 14 patients with cardiac, urological, orthopaedic, and general medicine related problems, who underwent treatment at the SSSIHMS-PG. Samples included 9 isolates from pus, 2 from urine, and 1 each from the pleural fluid, blood, and endotracheal tube (Table 1). All samples were cultured at the Department of Microbiology, SSSIHMS-PG. Only one isolate from each patient was included in the study.

\section{Background}

A 59-year-old male patient with a history of left main coronary artery disease underwent an operation in December 2015. Due to low ejection fraction, he was admitted to the intensive care unit post-operatively and required prolonged ventilator and inotropic support. In January 2016, blood culture from a central line catheter revealed the presence of colistin-resistant, MDR A. baumannii. To further understand the prevalence of $A$. baumannii among patients being treated at this centre, all $A$. baumannii infections reported over a 6-month period (October 2015 to March 2016) were analysed.

\section{Identification and Antibiotic Susceptibility Testing}

Organism identification and antibiotic susceptibility testing (AST) of the $14 \mathrm{~A}$. baumannii isolates was performed using VITEK 2.0 compact system and GN-281 AST cards (Table 2). Interpretation of the AST was done as per CLSI (Clinical Laboratory Standards Institute) guidelines [12]. Furthermore, glycerol stocks of the pure cultures were made and stored at $-80^{\circ} \mathrm{C}$ for future use. Based on the antibiotic resistance patterns, the 14 study isolates were categorized as AS, MDR, or XDR.

Whole-Genomic DNA Extraction and Quantification

Whole-genomic DNA from all the isolates was extracted from $3 \mathrm{~mL}$ of overnight LB broth culture by following the manufac- 
Table 1. Sources of sample collection

\begin{tabular}{|c|c|c|c|c|c|}
\hline Sample ID & Age & Gender & Source & Chief clinical complaint & Date of isolation \\
\hline $\mathrm{Ab} 1$ & 9 months & male & pus & congenital cyanotic heart disease & October 3, 2015 \\
\hline $\mathrm{Ab} 2$ & 65 years & male & urine & urological dysfunction & October 20, 2015 \\
\hline $\mathrm{Ab} 3$ & 20 years & female & pus & wound infection & October 21,2015 \\
\hline $\mathrm{Ab} 4$ & 10 months & male & pleural fluid & congenital cyanotic heart disease & October 28, 2015 \\
\hline $\mathrm{Ab} 5$ & 22 years & female & pus & wound infection & November 7, 2015 \\
\hline $\mathrm{Ab} 6$ & 32 years & male & pus & fracture wound & November 20,2015 \\
\hline $\mathrm{Ab} 8$ & 69 years & female & pus & fracture wound & December 14, 2015 \\
\hline $\mathrm{Ab} 11$ & 59 years & male & blood & coronary artery disease & January 10,2016 \\
\hline $\mathrm{Ab} 12$ & 8 years & female & urine & fracture wound & February 5, 2016 \\
\hline $\mathrm{Ab} 13$ & 25 years & male & pus & fracture wound & February 16, 2016 \\
\hline $\mathrm{Ab} 14$ & 59 years & male & pus & trauma wound & February 25, 2016 \\
\hline $\mathrm{Ab} 15$ & 57 years & male & pus & $\begin{array}{l}\text { chronic osteomyelitis; with pathological } \\
\text { subtrochanteric fracture }\end{array}$ & March 15, 2016 \\
\hline
\end{tabular}

turer's protocol (Machery-Nagel NucleoSpin-Tissue DNA extraction kit). Quantification of the DNA was done by NanoDrop, while the quality of DNA was assessed by agarose $(0.8 \%)$ gel electrophoresis.

\section{PCR Screening}

Gene sequences of virulence factors were obtained from the virulence factor database (VFDB- http://www.mgc.ac.cn/VFs/) and specific primers were designed for 10 virulence factors (ompA, bfmR, abaI, abaR, ptkA, plc, pld, hem $O$, adeF, and $p b p G)$. Screening of virulence genes, $A M R$ genes, AbaR islands (with or without disruption of the comM gene), and composite transposon Tn 1213 was performed as described previously $[11,13]$, or standardized in this study. Three primers were used to amplify (a) intact $\operatorname{com} M$ without any insertion, (b) initial transitional region comM-AbaR, and (c) final transitional region AbaR-comM. Primer details and PCR conditions are tabulated in online supplementary Tables 1 and 2 (see www.karger.com/doi/10.1159/000508461 for all online suppl. material), respectively. PCR was carried out using a final concentration of $0.5 \mu \mathrm{M}$ of each primer in a $20-\mu \mathrm{L}$ reaction. TaKaRa's SapphireAmp 2X PCR master mix was used for all the PCR amplifications.

\section{Random Amplified Polymorphic DNA-Based Phylogeny}

The clonal relationship among the 14 study isolates was studied. Twelve different random amplified polymorphic DNA (RAPD) primers (online suppl. Table 1) were used for PCR amplification, and the banding pattern from the primer which produced bands in all the isolates was further analysed by SYNGENE GeneTools software. A dendrogram was generated using the unweighted pair group method with arithmetic mean (UPGMA).

\section{Biofilm Formation}

Biofilm formation was investigated. Briefly, $100-\mu \mathrm{L}$ aliquots of bacterial culture $\left(\mathrm{OD}_{600}\right.$ of 0.1$)$ were inoculated in 96 -well roundbottom plates and incubated without shaking for $48 \mathrm{~h}$ at $37^{\circ} \mathrm{C}$. Following incubation, the plates were washed 3 times using $200 \mu \mathrm{L}$
$\mathrm{ddH}_{2} \mathrm{O}$, and then stained with $0.1 \%$ crystal violet ( $125 \mu \mathrm{L} /$ well) for $10 \mathrm{~min}$ at room temperature. The plates were then washed twice with $200 \mu \mathrm{L} \mathrm{ddH_{2 }} \mathrm{O}$ to remove excess crystal violet and dried overnight at room temperature. Solubilisation of the crystal violet was performed by adding $200 \mu \mathrm{L}$ of $30 \%$ acetic acid per well for $10 \mathrm{~min}$ at room temperature. Finally, $125 \mu \mathrm{L}$ of the solubilised mixture was transferred to a fresh flat-bottom 96-well plate and the biofilm production was quantified $\left(\mathrm{OD}_{595}\right)$ using a UV-Vis spectrophotometer. All experiments were conducted in triplicate [14].

\section{Results}

Fourteen A. baumannii isolates were collected from pus, blood, pleural fluid, endotracheal tube, and urine samples from 4 female and 10 male patients (Table 1) who underwent treatment at the SSSIHMS-PG. Nine of these isolates were obtained from patients with fractures (wound infections), 4 were isolated from patients with cardiac ailments, and 1 was from a patient with urological dysfunction. The age of the patients ranged from 9 months to 69 years. Thirteen of the isolates were from patients with community-acquired infections and 1 patient was found to have post-operative/nosocomial infection with colistin resistance (Ab11).

VITEK 2-based AST indicated that 71.4\% (10 isolates) were resistant to piperacillin/tazobactam; $78.57 \%$ (11 isolates) were resistant to ceftazidime and cefepime; $57.14 \%$ (8 isolates) were resistant to cefaperazone/sulbactum and levofloxacin; $64.28 \%$ (9 isolates) were resistant to doripenem, imipenem, ciprofloxacin and trimethoprim- /sulfamethoxazole; and 50\% (7 isolates) were resistant to 


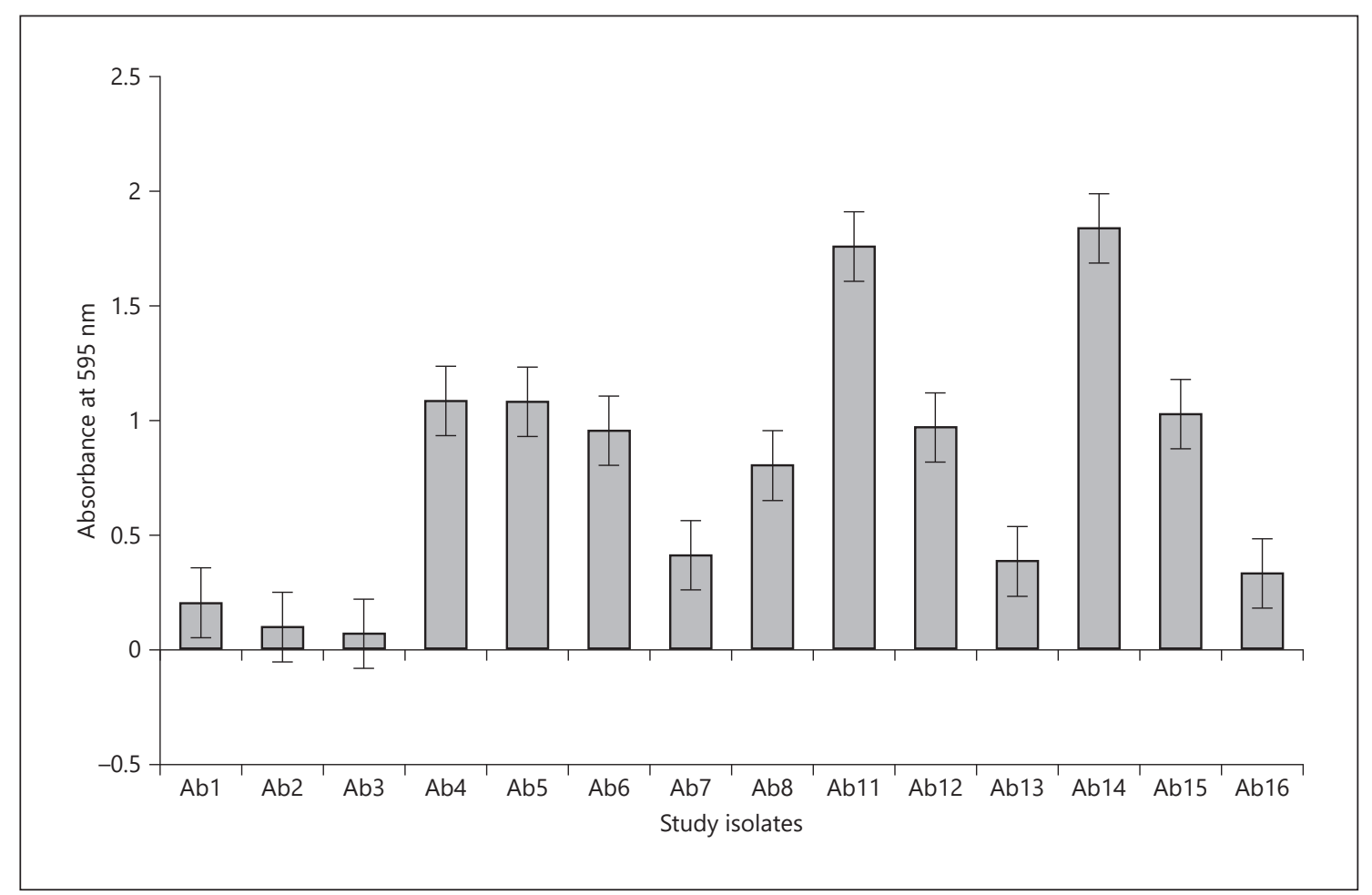

Fig. 1. Biofilm development (at $48 \mathrm{~h}$ ) on microtitre plate wells by clinical isolates of Acinetobacter baumannii.

gentamicin. Significantly, all isolates were found to be resistant to monobactam (Aztreonam) but sensitive to tigecycline (Table 2). Importantly, Ab14 and Ab11 were resistant to minocycline and colistin, respectively. Based on the antibiotic sensitivity patterns, the 14 isolates were categorized as AS (Ab15 and Ab16), MDR (Ab8, Ab11, and $\mathrm{Ab} 13$ ), and XDR (Ab1, Ab2, Ab3, Ab4, Ab5, Ab6, Ab7, $\mathrm{Ab} 12$ and $\mathrm{Ab} 14)$.

PCR screening of the 14 isolates suggested that they all contain at least 1 category of AMR-related gene (Table 3); $64.28 \%$ were positive for $b l a_{\mathrm{TEM}}, 14.28 \%$ ( 2 isolates) were positive for $b l a_{\mathrm{OXA}}$ and $b l a_{\mathrm{VIM}} ; 42.8 \%$ (6 isolates) were positive for $b l a_{\mathrm{NDM}}$; only 1 isolate was positive for $b l a_{\mathrm{SIM}}$, while $b l a_{\text {PER-1 }}$ was found in all isolates. sul1 was present in $85.7 \%$ (12) of the isolates and qacE1 in $71.42 \%(10)$ of the isolates. All the isolates were found to possess the Intl1 gene. None of the mobilizable colistin-resistance genes ( $m c r-1-m c r-5)$ were detected in the study isolates including Ab11.

Our investigations revealed the presence of $\mathrm{AbaR}$ islands in several of the study isolates. Screening of the isolates for AbaR islands and the comM ATPase gene revealed the presence of intact comM gene in 2 (Ab8 and $\mathrm{Ab16}$ ), while the presence of $\mathrm{AbaR}$ was confirmed in 10 of the study isolates. In the 4 remaining isolates (Ab8, $\mathrm{Ab} 14, \mathrm{Ab} 15$, and Ab16), our results did not confirm the presence or absence of $\mathrm{AbaR}$. Interestingly, Tn1213, a composite transposon, which is known to carry bla $a_{\text {PER-1 }}$ [10] was not found in any of the study isolates but all of them were found to possess bla $a_{\mathrm{PER}-1}$ (online suppl. Fig. 1-4).

PCR analyses for the presence of 10 selected virulence factor genes revealed that all 14 isolates were positive for ompA, adeF, abaI, bfmR, abaR, plc, and $p t k A$ virulence factors. $p b p G$ was present in 13 (92.8\%) of the study isolates, pld in $11(78.57 \%)$, and hemO in $10(71.4 \%)$. The presence of the $b f m R$ gene, which is involved in the production of biofilm, in all 14 isolates, suggested that the $A$. baumannii under study may be capable of biofilm production. Our investigations revealed that majority of the isolates (71.4\%) are strong biofilm producers (Fig. 1; online suppl. Fig. 5). Interestingly, the biofilm production potential of the AS Ab15 and Ab16 isolates was found to be higher than in the Ab1, Ab2, and Ab3 isolates which were characterized as XDR.

Phylogenetic analysis of the $14 \mathrm{~A}$. baumannii isolates under study by RAPD PCR suggested that they are unrelated and may have different clonal origins. In this regard, 
Fig. 2. RAPD profile of 14 A. baumannii

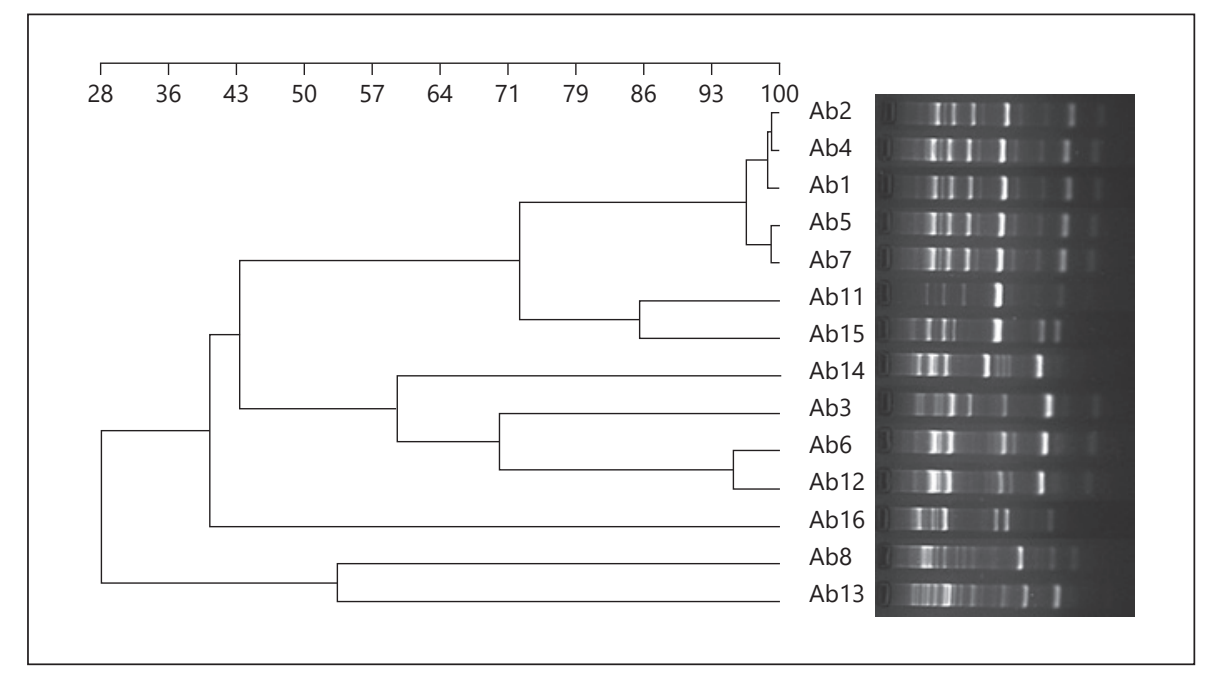

12 different primers were used to identify an appropriate primer for A. baumannii isolated from the Indian subcontinent. Primer OPA-3 was found to amplify and produce good banding patterns against all the tested isolates (Fig. 2). Phylogenetically, Ab11 (colistin-resistant A. baumannii) was not related to the other study isolates. However, its banding pattern indicated some relation to Ab15, which is phenotypically AS.

\section{Discussion}

Infections associated with $A$. baumannii are difficult to treat. With the increased emergence of MDR, XDR, and pan-drug-resistant (PDR) variants of this pathogen, limited therapeutic options are available to the treating clinicians. With its high population density and the easy access to antibiotics through their sale over the counter, the Indian subcontinent is a fertile ground for the spread of resistance determinants and the emergence of highly resistant bacterial phenotypes.

In this study, 12 isolates (85.7\%) were found to be antibiotic-resistant and only 2 were sensitive against most of the tested antibiotics. Phylogenetic analyses of RAPD suggested that the study isolates do not have a common origin. Our study findings correlate with previous reports on increased resistance against the fluoroquinolone [15] and $\beta$-lactam [16-18] classes of antibiotics among $A$. baumannii isolates; this rules out their use as a first-line drug. We found that $b l a_{\text {TEM }}$ is the most prevalent ESBL among A. baumannii isolates, although previous reports have indicated that $b l a_{\mathrm{CTX}-\mathrm{M}}$ is the pre- dominant ESBL gene $[19,20]$. Similar findings were observed in our previous report on carbapenem-resistant Enterobacteriaceae [8]. This study suggests that $b l a_{\mathrm{SHV}}$ is not a commonly encountered ESBL gene among $A$. baumannii strains on the Indian subcontinent. These results indicate the existence of variations in terms of prevalence and ESBL types in different geographical regions and demonstrate the diversity in the bacterial gene pool.

Generally, imipenem (carbapenems) and amikacin (aminoglycosides) are found to be the most active antimicrobials against A. baumannii [21]. However, $64 \%$ of the study isolates were found to be resistant to doripenem and imipenem antibiotics. This was confirmed by the presence of at least one carbapenemase/MBL (metallo- $\beta$-lactamase) genes in these isolates. This is in line with recent reports on increasing carbapenem resistance among bacteria [22]. bla $a_{\mathrm{OXA}}$ was identified in 2 isolates, $b l a_{\mathrm{NDM}}$ in $6, b l a_{\mathrm{VIM}}$ in 3 , and bla $\mathrm{SIM}_{\mathrm{S}}$ in 1 . As described previously, $b l a_{\mathrm{GIM}}$ and $b l a_{\mathrm{SPM}}$ were not identified in the study isolates. Our data represents a minor fraction of a hospital's meta-data and may not be representative of the current scenario. The presence of colistin resistance in the nosocomial isolate $\mathrm{Ab} 11$ reveals the complexity in dealing with pathogens resistant to the dwindling number of front-line drugs. It is imperative that these antibiotics of last resort are used judiciously before it is too late. The absence of any of the tested $\mathrm{mcr}$ genes [23] in the isolate Ab11 suggests that other mechanisms such as efflux pumps [24] and those which are yet to be described may have contributed to its emergence as colistin-resistant. 
The AbaR island integrated into comM [9] has been detected in these strains. We found that 12 isolates had AbaR integrated into the com $M$ gene, and 2 had intact comM gene in addition to AbaR integrated into the $\operatorname{com} M$ cassette. This indicates that there might be $>1$ copy of comM gene in these isolates and that 1 remained intact while the other was disrupted due to the integration of AbaR. Tn1213 is a composite transposon, which is inserted specifically between ISPa12 and ISPa13 [10]. Although our results suggested that $\operatorname{Tn} 1213$ was not present, bla $a_{\mathrm{PER}-1}$, commonly associated with Tn1213, was found to be prevalent in all $14 \mathrm{~A}$. baumannii isolates, suggesting that multiple mechanisms are involved in the dissemination of bla $a_{\mathrm{PER}-1}$. Many of the class 1 integron genes identified from clinical settings reveal modifications to their ancestral composition including the incorporation of sulfonamide resistance gene (sul1) and/or the deletion of qacE1 (a determinant of quaternary ammonium compound resistance). In our study, all 14 isolates were positive for the Intl1 gene, 12 were positive for sul1, and 10 were positive for qacE1. The presence of qacE1 among the study isolates, a gene commonly associated with the class 1 integron, which provides resistance to quaternary ammonium compounds, correlates with the increased use of detergents and disinfectants in hospital settings. The rapid dissemination of MGEs possessing antibiotic resistance and virulence factor gene cassettes may have been aided by the increase in global travel [25]. This is exacerbated by the capacity of the pathogens to produce biofilms, which poses a significant clinical challenge in the treatment of hospitalized patients.

\section{Conclusion}

This study presents the molecular characteristics of clinically isolated, pathogenic, and antibiotic-resistant $A$. baumannii from a tertiary care hospital in South India. The prevalence of MDR and XDR pathogens including those resistant to colistin, although limited in number, is an alarming situation that needs urgent and drastic interventions. Indiscriminate use of antibiotics may be a very strong selection pressure for the maintenance and spread of several resistance determinants among the clinical isolates. The presence of MGEs further compounds the already deteriorating situation due to the lack of availability of newer classes of antibiotics and therapeutic agents. Implementation of the current national and international guidelines in combatting these dangerous pathogens assumes critical proportions due to their extreme pathogenic potential, including those which were found to be AS. In this regard, devising and incorporating appropriate infection control measures, together with robust antibiotic stewardship programs, is recommended.

\section{Acknowledgements}

We acknowledge UGC-SAP (DRS), DST-FIST and DBT-BIF, Government of India for the infrastructural support to the Department of Biosciences, SSSIHL, Prasanthi Nilayam and UGC SRF, ICMR-SRF, and NFST Fellowships from the Government of India to B.K., C.P.P., and M.L.

\section{Conflict of Interest Statement}

There were no conflicts of interest.

\section{References}

1 Aly NY, Al-Mousa HH, Al Asar SM. Nosocomial infections in a medical-surgical intensive care unit. Med Princ Pract. 2008;17(5):373-7.

2 King LB, Swiatlo E, Swiatlo A, McDaniel LS. Serum resistance and biofilm formation in clinical isolates of Acinetobacter baumannii. FEMS Immunol Med Microbiol. 2009 Apr; 55(3):414-21.

3 Longo F, Vuotto C, Donelli G. Biofilm formation in Acinetobacter baumannii. New Microbiol. 2014 Apr;37(2):119-27.

4 Willyard C. The drug-resistant bacteria that pose the greatest health threats. Nature. 2017 Feb;543(7643): 15.

5 Sudhaharan S, Vemu L, Kanne P. Prevalence of multidrug resistant Acinetobacter baumannii in clinical samples in a tertiary care hospital. Int J Infect Control. 2015;11(3).
6 He X, Lu F, Yuan F, Jiang D, Zhao P, Zhu J, et al. Biofilm formation caused by clinical Acinetobacter baumannii isolates is associated with overexpression of the AdeFGH efflux pump. Antimicrob Agents Chemother. 2015 Aug;59(8):4817-25.

7 Durante-Mangoni E, Zarrilli R. Global spread of drug-resistant Acinetobacter baumannii: molecular epidemiology and management of antimicrobial resistance. Future Microbiol. 2011 Apr;6(4):407-22.

8 Mahalingam N, Manivannan B, Khamari B, Siddaramappa S, Adak S, Bulagonda EP. Detection of Antibiotic Resistance Determinants and Their Transmissibility among Clinically Isolated Carbapenem-Resistant Escherichia coli from South India. Med Princ Pract. 2018; 27(5):428-35.
9 Douraghi M, Jasemi S, Kodori M, Rahbar M, Boroumand MA. Evidence of Interruption of the comM Gene in a Large Series of Clinical Isolates of Multidrug-Resistant Acinetobacter baumannii. J Mol Microbiol Biotechnol. 2016;26(6):410-3.

10 Mancini S, Poirel L, Kieffer N, Nordmann P. Transposition of Tn1213 Encoding the PER-1 Extended-Spectrum $\beta$-Lactamase. Antimicrob Agents Chemother. 2018 Feb;62(3): e02453-17.

11 Danel F, Hall LM, Gur D, Akalin HE, Livermore DM. Transferable production of PER-1 $\beta$-lactamase in Pseudomonas aeruginosa. J Antimicrob Chemother. 1995 Feb;35(2):28194.

12 CLSI. Performance standards for Antimicrobial susceptibility Testing. Wayne (PA): Clinical and Laboratory Standards Institute; 2019. 
13 Post V, White PA, Hall RM. Evolution of AbaR-type genomic resistance islands in multiply antibiotic-resistant Acinetobacter baumannii. J Antimicrob Chemother. 2010 Jun; 65(6):1162-70.

14 O'Toole GA. Microtiter dish biofilm formation assay. J Vis Exp. 2011 Jan;(47):2437.

15 Park S, Lee KM, Yoo YS, Yoo JS, Yoo JI, Kim HS, et al. Alterations of gyrA, gyrB, and parC and Activity of Efflux Pump in Fluoroquinolone-resistant Acinetobacter baumannii. Osong Public Health Res Perspect. 2011 Dec; 2(3):164-70.

16 Paton R, Miles RS, Hood J, Amyes SG, Miles RS, Amyes SG. ARI 1: $\beta$-lactamase-mediated imipenem resistance in Acinetobacter baumannii. Int J Antimicrob Agents. 1993 Feb; 2(2):81-7.

17 Ling TK, Ying CM, Lee CC, Liu ZK. Comparison of antimicrobial resistance of Acinetobacter baumannii clinical isolates from Shanghai and Hong Kong. Med Princ Pract. 2005 Sep-Oct;14(5):338-41.
18 Maragakis LL, Perl TM. Acinetobacter baumannii: epidemiology, antimicrobial resistance, and treatment options. Clin Infect Dis. 2008 Apr;46(8):1254-63.

19 Kaur M, Aggarwal A. Occurrence of the CTXM, SHV and the TEM Genes Among the Extended Spectrum $\beta$-Lactamase Producing Isolates of Enterobacteriaceae in a Tertiary Care Hospital of North India. J Clin Diagn Res. 2013 Apr;7(4):642-5.

20 Bajpai T, Pandey M, Varma M, Bhatambare GS. Prevalence of TEM, SHV, and CTX-M Beta-Lactamase genes in the urinary isolates of a tertiary care hospital. Avicenna J Med. 2017 Jan-Mar;7(1):12-6.

21 Al-Tawfiq JA, Antony A, Abed MS. Antimicrobial resistance rates of Enterobacter spp.: a seven-year surveillance study. Med Princ Pract. 2009;18(2):100-4.
22 Kazemian H, Heidari H, Ghanavati R, Ghafourian S, Yazdani F, Sadeghifard N, et al. Phenotypic and genotypic characterization of ESBL-, AmpC-, and carbapenemase-producing Klebsiella pneumoniae and Escherichia coli isolates. Med Princ Pract. 2019;28(6): 547-51.

23 Liu YY, Wang Y, Walsh TR, Yi LX, Zhang R, Spencer J, et al. Emergence of plasmid-mediated colistin resistance mechanism MCR-1 in animals and human beings in China: a microbiological and molecular biological study. Lancet Infect Dis. 2016 Feb;16(2):161-8.

24 Harding CM, Hennon SW, Feldman MF. Uncovering the mechanisms of Acinetobacter baumannii virulence. Nat Rev Microbiol. 2018 Feb;16(2):91-102.

25 Senok AC, Botta GA, Soge OO. Emergence and spread of antimicrobial-resistant pathogens in an era of globalization. Interdiscip Perspect Infect Dis. 2012;2012:286703. 